\title{
Salivary cortisol is not associated with incident insulin resistance or type 2 diabetes mellitus
}

\author{
Karim Gariani' ${ }^{1}$, Pedro Marques-Vidal ${ }^{2}$, Gérard Waeber ${ }^{2}$, Peter Vollenweider ${ }^{2}$ and François R Jornayvaz ${ }^{1}$ \\ 'Service of Endocrinology, Diabetes, Hypertension and Nutrition, Geneva University Hospitals, Geneva, Switzerland \\ 2Departments of Medicine and Internal Medicine, Lausanne University Hospital, Lausanne, Switzerland \\ Correspondence should be addressed to F R Jornayvaz: francois.jornayvaz@hcuge.ch
}

\begin{abstract}
Background: Excessive glucocorticoid secretion has been associated with type 2 diabetes mellitus (T2DM) and other features of the metabolic syndrome. We aimed to evaluate whether basal or evening salivary cortisol may predict the occurrence of incident insulin resistance (IR) or T2DM.

Method: This was a prospective, population-based study derived from the CoLaus/ PsyCoLaus study including 1525 participants (aged $57.7 \pm 10.3$ years; 725 women). A total of 1149 individuals were free from T2DM at baseline. Fasting plasma glucose and insulin were measured after a follow-up of 5.3 years. Basal and evening salivary cortisol were measured at baseline. The association between basal or evening salivary cortisol level and incidence of IR or T2DM were analyzed by logistic regression, and the results were expressed for each independent variable as ORs and $95 \% \mathrm{Cl}$.

Results: After a median follow-up of 5.3 years, a total of 376 subjects (24.7\%) developed IR and 32 subjects (2.1\%) developed T2DM. Basal and evening salivary cortisol divided in quartiles were not associated with incidence of IR or T2DM. Multivariable analysis for age, gender, body mass index, physical activity and smoking status showed no association between basal or evening salivary cortisol and incidence of IR or T2DM. Conclusion: In the CoLaus/PsyCoLaus study of healthy adults, neither basal nor evening salivary cortisol was associated with incident IR or T2DM.
\end{abstract}

\author{
Key Words \\ - salivary cortisol \\ - insulin resistance \\ - diabetes \\ - prospective study \\ - epidemiology
}

\section{Introduction}

Type 2 diabetes mellitus (T2DM) pathogenesis may involve the hypothalamic-pituitary-adrenal axis (HPA) (1). Common features observed in T2DM and metabolic syndrome, such as elevated fasting glucose, obesity, hyperlipidemia or hypertension, might be linked with an excess of glucocorticoid secretion (2, 3, 4). Patients with metabolic syndrome and concomitantly higher fasting cortisol have been related with more prevalent central obesity and hypertension, a higher triglycerides (TG) level, increased insulin resistance (IR) and a higher fasting plasma glucose (5). Patients with T2DM may exhibit evidence of subclinical hypercortisolism such as higher 24-h urine-free cortisol levels, higher plasma cortisol levels after dexamethasone suppression test and higher basal plasma cortisol levels compared to people not having T2DM $(6,7,8,9,10,11,12)$. Similarly, Cushing syndrome, which is characterized by an excessive endogenous glucocorticoid production, is associated with IR and cardiovascular risk factors (13). Several studies have shown that individuals with glucose intolerance or T2DM exhibit a significantly higher level of chronic stress and hormonal stress responses (14, 15). Salivary cortisol has been established as a reliable indicator of circulating cortisol concentrations and HPA axis function (16). Moreover studies assessing stress-linked perturbation of HPA axis have proposed that sustained-activated HPA axis and subsequent cortisol increase might be associated with features of the metabolic syndrome $(17,18)$. 
Furthermore, in patients with T2DM, increased HPA activity is associated with the presence of diabetes complications, and the level of cortisol secretion is linked to the number of diabetes complications (19).

Cortisol secretion follows a circadian rhythm with a nadir at midnight, starts to rise at 02:00-04:00 h, displays a peak briefly after waking up and then shows a progressive decrease during the day (20). The normal circadian rhythm is disturbed in Cushing syndrome (21). Salivary cortisol can be used instead of serum total cortisol, since it represents the free fraction of cortisol, which is the active form (4). Prospective studies evaluating the link between cortisol secretion using salivary cortisol and new onset of T2DM remain limited. In the Longitudinal Aging Study of Amsterdam (LASA), elevated evening salivary cortisol was associated with future T2DM development in women but not in men (22). In the Whitehall II Study, T2DM was related to a significantly flatter slope in salivary cortisol decline across the day and a greater bedtime salivary cortisol (18). In the same cohort, new onset of impaired fasting glucose (IFG) and T2DM was associated with a trend toward a flattened diurnal cortisol slope (23).

The aim of this study was therefore to assess the predictive value of basal and evening salivary cortisol on the incidence of IR and T2DM at 5.3 years, in a prospective population-based study conducted in healthy adults aged 50-75 years in Lausanne (Switzerland).

\section{Materials and methods}

\section{Subjects recruitment}

The CoLaus/PsyCoLaus (Cohorte lausannoise) study was designed to evaluate the prevalence of cardiovascular risk factors and to identify new molecular determinants of these risk factors in healthy adults living in the city of Lausanne (Switzerland). The study was approved by the Institutional Ethics Committee of the University of Lausanne and all participants provided written informed consent. The sampling procedure of the CoLaus/ PsyCoLaus study has been described previously $(24,25)$. Briefly, a simple, non-stratified random sample of 19,830 individuals of the city of Lausanne was drawn. The inclusion criteria were (a) written informed consent; (b) willingness to take part in the examination and to provide blood samples and (c) age between 35 and 75 years. Recruitment started in June 2003 and ended in May 2006 and included a total of 6733 participants. The assessment integrated an anamnesis, a physical examination, blood analysis and a set of questionnaires. The first follow-up visit was conducted between April 2009 and September 2012, 5.6 years on average after the collection of baseline data. The second follow-up was conducted between May 2014 and April 2017, 10.9 years on average after the baseline. Salivary cortisol was collected only in the follow-ups; hence, for this study, the first follow-up was considered as the baseline period.

\section{Salivary cortisol}

Saliva samples were taken using cotton swabs ('Salivette', Sarstedt, Germany). Each participant performed a salivary sample collection on waking up and in the evening. Subjects were informed not to brush their teeth and to avoid eating, drinking, smoking and exercising half an hour before the salivary sample collection (26). Subjects stored the saliva samples at home in their freezers before returning them to the laboratory together with the saliva protocol, where they were stored at $-20^{\circ} \mathrm{C}$ until biochemical analysis. Free urinary cortisol, ACTH or cortisolemia were not assessed.

Freecortisollevelsin the salivary samplesweremeasured using a commercially available chemiluminescence assay (IBL, Hamburg, Germany). Inter-assay and intra-assay coefficients of variability were $<9 \%$.

\section{Biological data collection}

Venous blood samples $(50 \mathrm{~mL})$ were collected in the fasting state. The majority of biological plasma measurements were performed at the clinical laboratory of the Lausanne University Hospital (CHUV). Glucose level was measured by glucose dehydrogenase with a maximum interassay $\mathrm{CV}$ of $2.1 \%$ and a maximum intra-assay $\mathrm{CV}$ of $1.0 \%$. Insulin was evaluated by a solid-phase, two-site chemiluminescent immunometric assay (Diagnostic Products Corporation) with a maximum intra-assay $\mathrm{CV}$ of $13.7 \%$. HDL cholesterol was measured by cholesterol oxidase-phenol-aminophenazone + polyethylene glycol+cyclodextrin with a maximum inter-assay $\mathrm{CV}$ of $3.6 \%$ and a maximum intra-assay CV of $0.9 \%$. TGs were assessed by GPO-PAP with a maximum inter-assay $\mathrm{CV}$ of $2.9 \%$ and a maximum intra-assay CV of $1.5 \%$.

Dyslipidemia was defined as TG concentration $>2.82 \mathrm{mmol} / \mathrm{L} \quad(250 \mathrm{mg} / \mathrm{dL})$ or a HDL cholesterol $<0.9 \mathrm{mmol} / \mathrm{L} \quad(35 \mathrm{mg} / \mathrm{dL})$, which are indications for testing for diabetes on the basis of the American Diabetes Association recommendations (27). T2DM was defined by a fasting glucose $\geq 7.0 \mathrm{mmol} / \mathrm{L}$ and/or if the participant reported having an anti-diabetic treatment. Fasting plasma 
glucose and insulin were measured, and the homeostasis model assessment index of IR (HOMA) was calculated according to Matthews et al. (28) Insulin resistance was defined by a HOMA index $>2.6$ (29).

\section{Other covariates}

All individuals had a visit in the morning after an overnight fast (minimum fasting time: $8 \mathrm{~h}$ ). Participants were asked about their personal and family history of cardiovascular disease and cardiovascular risk factors as well as their treatment.

Subjects were considered as smokers if they currently smoked, irrespective of the amount of duration; former smokers were considered irrespective of the time since quitting smoking; never smokers were defined as subjects, who never smoked.

Physical activity was evaluated by a set of questions (30) previously validated in the population of Geneva. This self-reported questionnaire evaluated the type and duration of 70 kinds of (non) professional activities and sports during the previous week. Total daily energy expenditure was computed and participants were categorized into tertiles of physical activity.

Body weight and height were measured with participants standing without shoes in light indoor clothes. Body weight was measured in kilograms to the nearest $100 \mathrm{~g}$ using a Seca scale (Seca $\mathrm{GmbH}$ ), which was calibrated regularly. Height was measured to the nearest $5 \mathrm{~mm}$ by using a Seca height gauge. BMI was computed as weight in $\mathrm{kg}$ divided par height in $\mathrm{m}^{2}$.

Blood pressure was measured three times on the left arm, with an appropriately sized cuff, after a rest of at least $10 \mathrm{~min}$ in the seated position with the use of an Omron HEM-907 automated oscillometric sphygmomanometer (Omron Healthcare). The average of the last two measurements was used for analyses. Hypertension was defined as a systolic blood pressure $\geq 140 \mathrm{mmHg}$ and/or a diastolic blood pressure $\geq 90 \mathrm{mmHg}$ and/or presence of antihypertensive drug treatment.

\section{Exclusion criteria}

Participants were excluded if they (1) had T2DM at inclusion; (2) did not participate in salivary sampling; (3) did not participate in the follow-up; (4) had any missing data in smoking status, bodyweight, height, hypertension, dyslipidemia or history of cardiovascular disease at baseline or (5) had missing data regarding T2DM or IR at follow-up.

\section{Statistical analysis}

Statistical analyses were conducted using Stata version 15.1 (Stata Corp.) for Windows. Descriptive results were expressed as number of participants (percentage) for categorical variables and as average \pm s.D. for continuous variables. Bivariate comparisons were performed using chi-square for categorical variables or Student's $t$-test for continuous variables. As it was not possible to obtain the precise data of the conversion into T2DM, the association between quartiles of salivary cortisol and incident T2DM or IR was modeled using logistic regression. Results were expressed as odds ratio (OR) and (95\% confidence interval) using the first quartile as reference. Two multivariable models were applied: the first one adjusted on age and gender; the second adjusted on age, gender, BMI, physical activity and smoking status. Testing for trend (linear, quadratic and cubic) regarding the effect of quartiles of salivary cortisol was performed using the contrast $\mathrm{p}$. command of Stata.

A sensitivity analysis was performed to take into account the fact that excluded and included participants differed significantly regarding several clinical and demographic characteristics. First, the probability of participation was computed using a logistic model with gender, age and BMI categories, smoking, tertiles of physical activity, hypertension, dyslipidemia and personal history of CVD as

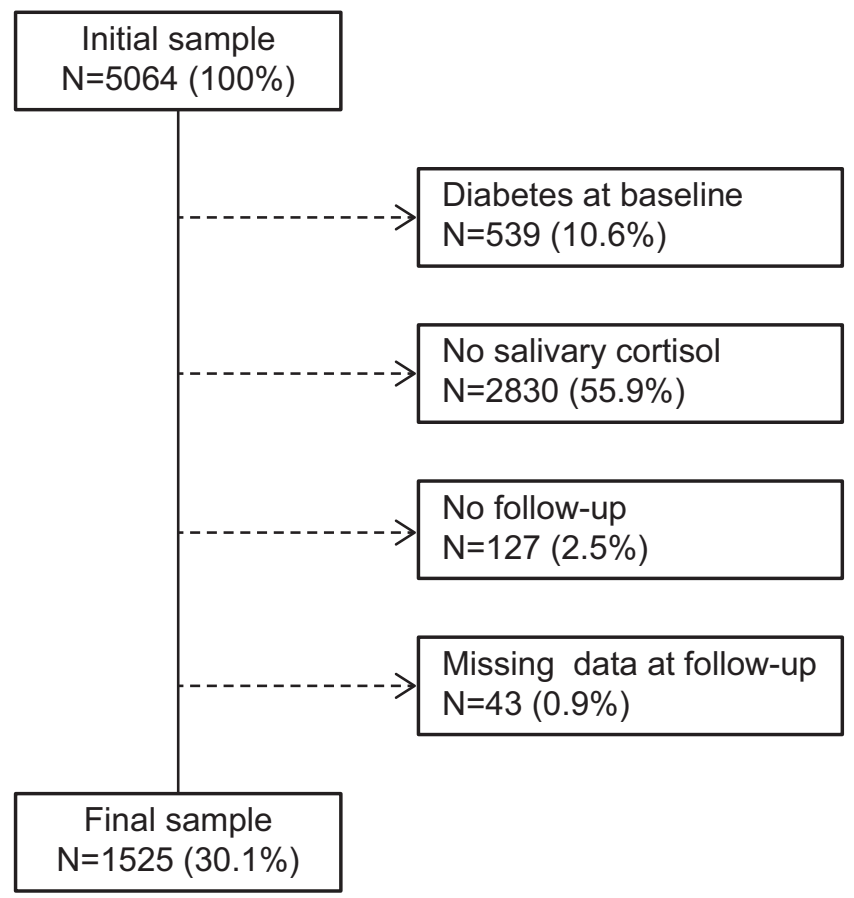

Figure 1

Included and excluded participants.

This work is licensed under a Creative Commons Attribution-NonCommercial-NoDerivatives 4.0 Internationab ticense. $i$ ica com at $04 / 26 / 2023$ 02:12:10AM 
the independent variables. The inverse of the probability of participation was used for weighting (31).

Statistical significance was considered for a two-sided test with $P<0.05$.

\section{Ethics, consent and permissions}

The Institutional Ethics Committee of the University of Lausanne, which afterward became the Ethics Commission of Canton Vaud (www.cer-vd.ch) approved the baseline CoLaus/PsyCoLaus study (reference 16/03), the first (reference 33/09) and the second (reference 26/14) follow-ups. The study was performed in agreement with the Helsinki Declaration and its former amendments, and in accordance with the applicable Swiss legislation. All participants gave their signed informed consent before entering the study.

\section{Results}

\section{Characteristics of participants}

Of the initial 5064 participants, 1525 (30.1\%) were retained for analysis. The reasons for exclusion are indicated in Fig. 1 and the characteristics of the included and the excluded participants are summarized in Supplementary
Table 1 (see section on supplementary data given at the end of this article). Excluded patients from the analysis were more often male, overweight, smokers and had more often hypertension or dyslipidemia (Supplementary Table 1).

\section{Associations between salivary cortisol and incident IR or diabetes mellitus}

After a follow-up of 5.3 years, respectively 376 (24.7\%) and 32 participants $(2.1 \%)$ of the 1525 included participants developed IR or T2DM. Participants who developed IR were more often male, older, overweight or obese, sedentary, had more often hypertension and dyslipidemia as well as a positive history of cardiovascular disease (Table 1). Participants who developed T2DM were more often overweight or obese, had more dyslipidemia, but there was no age or sex difference (Table 2). Smoking was associated with neither IR nor T2DM development (Tables 1 and 2). Basal salivary cortisol divided in quartiles showed no association between cortisol level and incidence of IR or T2DM (Table 3). After adjusting for age and gender, no association was found between basal salivary cortisol and incident IR or T2DM. A second multivariable model adding BMI, physical activity and smoking status as adjusted variables showed no association between basal

Table 1 Characteristics of healthy participants who developed and who remained free from insulin resistance ${ }^{a}$ at 5.3 years average follow-up.

\begin{tabular}{|c|c|c|c|}
\hline Insulin resistance & No & Yes & P value \\
\hline$n$ & 1149 & 376 & \\
\hline Women, $n(\%)$ & $725(63.1)$ & $187(49.7)$ & $<0.001$ \\
\hline Age, years & $57.0 \pm 10.1$ & $59.9 \pm 10.7$ & $<0.001$ \\
\hline $\mathrm{BMl}, \mathrm{kg} / \mathrm{m}^{2}$ & $24.3 \pm 3.5$ & $28.5 \pm 4.2$ & $<0.001$ \\
\hline BMI status, $n(\%)$ & & & $<0.001$ \\
\hline Normal & $720(62.7)$ & $64(17.0)$ & \\
\hline Overweight & $356(31.0)$ & $207(55.1)$ & \\
\hline Obese & $73(6.4)$ & 105 (27.9) & \\
\hline Smoking status, $n(\%)$ & & & 0.085 \\
\hline Never & 539 (46.9) & $153(40.7)$ & \\
\hline Former & $439(38.2)$ & $155(41.2)$ & \\
\hline Current & $171(14.9)$ & $68(18.1)$ & \\
\hline Physical activity tertiles, $n(\%)$ & & & $<0.001$ \\
\hline First & 451 (39.3) & $102(27.1)$ & \\
\hline Second & $391(34.0)$ & $140(37.2)$ & \\
\hline Third & 307 (26.7) & $134(35.6)$ & \\
\hline Sedentary status, $n(\%)$ & $604(52.6)$ & $239(63.6)$ & $<0.001$ \\
\hline Hypertension, n (\%) & $349(30.4)$ & $216(57.5)$ & $<0.001$ \\
\hline Dyslipidemia, n (\%) & $192(16.7)$ & $125(33.2)$ & $<0.001$ \\
\hline History of cardiovascular disease, $n(\%)$ & $28(2.4)$ & $29(7.7)$ & $<0.001$ \\
\hline
\end{tabular}

Results are expressed as average standard deviation for continuous variables or as number of participants (percentage) for categorical variables. Comparison between included and excluded participants performed using Student's $t$-test for continuous variables and chi-square for categorical variables.

ansulin resistance defined as a HOMA index $>2.6$.

https://ec.bioscientifica.com https://doi.org/10.1530/EC-19-0251 (c) 2019 The authors Published by Bioscientifica Ltd

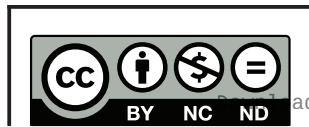

This work is licensed under a Creative Commons Attribution-NonCommercial-NoDerivatives 4.0 Internationab ticense.ifica.com at 04/26/2023 02:12:10AM 
Table 2 Characteristics of healthy participants who developed and who remained free from diabetes mellitus at 5.3 years average follow-up.

\begin{tabular}{l}
\hline Diabetes mellitus \\
\hline$n$ \\
Women, $n$ (\%) \\
Age, years \\
BMl, kg/m² \\
BMI status, $n$ (\%) \\
$\quad$ Normal \\
Overweight \\
Obese \\
Smoking status, $n(\%)$ \\
Never \\
Former \\
Current \\
Physical activity tertiles, $n$ (\%) \\
$\quad$ First \\
Second \\
Third \\
Sedentary status, $n(\%)$ \\
Hypertension, $n(\%)$ \\
Dyslipidemia, $n$ (\%) \\
History of cardiovascular disease, $n(\%)$
\end{tabular}

\begin{tabular}{c}
\hline No \\
\hline 1493 \\
$896(60.0)$ \\
$57.7 \pm 10.3$ \\
$25.3 \pm 4.1$ \\
$776(52.0)$ \\
$549(36.8)$ \\
$168(11.3)$ \\
$678(45.4)$ \\
$583(39.1)$ \\
$232(15.5)$ \\
$543(36.4)$ \\
$518(34.7)$ \\
$432(28.9)$ \\
$820(54.9)$ \\
$545(36.5)$ \\
$306(20.5)$ \\
$55(3.7)$
\end{tabular}

\begin{tabular}{|c|c|}
\hline Yes & $P$ value \\
\hline \multicolumn{2}{|l|}{32} \\
\hline $16(50.0)$ & 0.253 \\
\hline $61.1 \pm 10.5$ & 0.065 \\
\hline \multirow{2}{*}{$28.1 \pm 4.8$} & $<0.001$ \\
\hline & $<0.001$ \\
\hline \multicolumn{2}{|l|}{$8(25.0)$} \\
\hline \multicolumn{2}{|l|}{$14(43.8)$} \\
\hline \multicolumn{2}{|l|}{10 (31.3) } \\
\hline & 0.607 \\
\hline \multicolumn{2}{|l|}{$14(43.8)$} \\
\hline \multicolumn{2}{|l|}{$11(34.4)$} \\
\hline \multicolumn{2}{|l|}{7 (21.9) } \\
\hline & 0.760 \\
\hline \multicolumn{2}{|l|}{$10(31.3)$} \\
\hline \multicolumn{2}{|l|}{13 (40.6) } \\
\hline \multicolumn{2}{|l|}{9 (28.1) } \\
\hline 23 (71.9) & 0.056 \\
\hline $20(62.5)$ & 0.003 \\
\hline $11(34.4)$ & 0.056 \\
\hline $2(6.3)$ & 0.449 \\
\hline
\end{tabular}

Results are expressed as average standard deviation for continuous variables or as number of participants (percentage) for categorical variables. Comparison between included and excluded participants performed using Student's $t$-test for continuous variables and chi-square for categorical variables.

salivary cortisol and new onset of IR or T2DM (Table 4). Similarly, evening salivary cortisol divided in quartiles showed no association between cortisol level and incidence of IR or T2DM (Table 5), and this remained true after multiple adjustments (Table 6).

Because of the low incidence of IR and T2DM, we performed a multivariable analysis of the association between quartiles of salivary cortisol and incident IR and T2DM at 5.3 years average follow-up, using inverse probability weighting. This analysis confirmed the absence of significant association between basal salivary cortisol and incident IR or T2DM (Supplementary Table 2), and between evening salivary cortisol and incident IR or T2DM (Supplementary Table 3). Finally, bi and multivariable analysis did not show any significant association between basal or evening salivary cortisol quartiles and BMI, fasting plasma glucose or insulinemia (Supplementary Table 4).

\section{Discussion}

To our knowledge, this is one of the largest prospective studies assessing the association between salivary cortisol and incident IR or T2DM. Our results show that neither

Table 3 Distribution between quartiles of basal salivary cortisol $(\mathrm{nmol} / \mathrm{L})$ of healthy participants who developed $(n=376)$ and who remained free $(n=1149)$ from insulin resistance ${ }^{a}$ and remained free $(n=1493)$ and developed diabetes mellitus $(n=32)$ at 5.3 years average follow-up ${ }^{\mathrm{b}}$.

\begin{tabular}{|c|c|c|c|c|c|}
\hline & First & Second & Third & Fourth & $P$ value \\
\hline Salivary cortisol, median (range) & $9.4(0.5-12.5)$ & $14.8(12.5-17.1)$ & $20.1(17.1-23.9)$ & $29.4(23.9-68.6)$ & 0.971 \\
\hline No insulin resistance, $n(\%)$ & $286(74.9)$ & $288(75.6)$ & $285(74.8)$ & $290(76.1)$ & \\
\hline Incident insulin resistance, $n(\%)$ & $96(25.1)$ & $93(24.4)$ & $96(25.2)$ & $91(23.9)$ & \\
\hline Salivary cortisol, median (range) & $9.4(0.5-12.5)$ & $14.8(12.5-17.1)$ & $20.1(17.1-23.9)$ & $29.4(23.9-68.6)$ & 1.000 \\
\hline No diabetes mellitus, $n(\%)$ & $374(97.9)$ & $373(97.9)$ & $373(97.9)$ & $373(97.9)$ & \\
\hline Diabetes mellitus, $n(\%)$ & $8(2.1)$ & $8(2.1)$ & $8(2.1)$ & $8(2.1)$ & \\
\hline
\end{tabular}

Statistical analysis by chi-square; the $P$ value corresponds to the chi-square comparing the distribution of participants with and without incident insulin resistance between quartiles of the different variables. $n=1493$ no diabetes mellitus and $n=32$ diabetes mellitus.

ansulin resistance defined by a HOMA index $>2.6, n=1149$ no insulin resistance and $n=376$ incident insulin resistance. ${ }^{b}$ Results are expressed as number of participants (percentage).

https://ec.bioscientifica.com https://doi.org/10.1530/EC-19-0251 (c) 2019 The authors Published by Bioscientifica Ltd

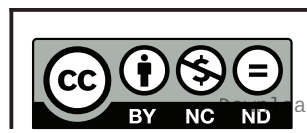

This work is licensed under a Creative Commons Attribution-NonCommercial-NoDerivatives 4.0 Internationab sicense.ifica . com at $04 / 26 / 2023 \quad 02: 12: 10 \mathrm{AM}$ 
Table 4 Multivariable analysis of the association between quartiles of basal salivary cortisol and incident insulin resistance and diabetes mellitus at 5.3 years average follow-up.

\begin{tabular}{l}
\hline Incident insulin resistance \\
Cases (\%) \\
Multivariable model 1 \\
Multivariable model 2 \\
Diabetes mellitus \\
Cases (\%) \\
Multivariable model 1 \\
Multivariable model 2 \\
\hline
\end{tabular}

\begin{tabular}{c}
\hline First \\
\hline 96 (25.1) \\
1 (ref) \\
1 (ref) \\
\\
$8(2.1)$ \\
1 (ref) \\
1 (ref) \\
\hline
\end{tabular}

\begin{tabular}{c}
\hline Second \\
\hline $93(24.4)$ \\
$0.96(0.69-1.34)$ \\
$1.01(0.70-1.45)$ \\
$8(2.1)$ \\
$0.99(0.37-2.69)$ \\
$1.00(0.37-2.73)$ \\
\hline
\end{tabular}

\begin{tabular}{c}
\hline Third \\
\hline $96(25.2)$ \\
$0.94(0.67-1.31)$ \\
$1.01(0.70-1.45)$ \\
$8(2.1)$ \\
$0.94(0.35-2.54)$ \\
$0.96(0.35-2.61)$ \\
\hline
\end{tabular}

\begin{tabular}{c}
\hline Fourth \\
\hline $91(23.9)$ \\
$0.90(0.65-1.26)$ \\
$1.05(0.73-1.52)$ \\
$8(2.1)$ \\
$0.97(0.36-2.62)$ \\
$1.08(0.40-2.95)$ \\
\hline
\end{tabular}
P value

Insulin resistance was defined by a HOMA index >2.6. $n=1149$ no insulin resistance and $n=376$ incident insulin resistance. $n=1493$ no diabetes mellitus and $n=32$ diabetes mellitus. Statistical analysis was conducted by logistic regression and results are expressed as odds ratio ( $95 \%$ confidence interval). Multivariable model 1: adjusting on age and gender. Multivariable model 2: adjusting on age, gender, BMI, physical activity and smoking status.

basal nor evening salivary cortisol is associated with newonset IR or T2DM.

Salivary cortisol measurement may represent an attractive way to evaluate glucocorticoid excess. Salivary cortisol has some advantages compared to blood or urine measurements. Salivary cortisol is noninvasive, whereas blood sampling could be stressful and artificially increase cortisol levels. Individuals can collect samples in their normal environment. Urine collections have the disadvantage of requiring a 24-h collection. However, salivary cortisol has some limitations. Notably, it varies diurnally, displays an inconstant awakening response and daily acute fluctuations $(32,33)$.

In this study, we could not show an association between basal or evening salivary cortisol and risk of future IR or T2DM. In the Whitehall II Study, six samples were taken over the course of a normal weekday allowing assessing the cortisol awaking response, the slope across the day and the bedtime cortisol (23). Interestingly, the authors did not observe any significant association between these different cortisol measurements and incidence of T2DM, which is in concordance with our findings using basal and evening salivary cortisol measurements. In the LASA cohort, only older individuals ( $\geq 65$ years) were included and salivary cortisol was taken in the morning and in the evening. In this study, the authors found an association between salivary cortisol and risk of new T2DM only with the evening salivary cortisol in women (OR 1.33, $P=0.01$ ). Evening cortisol in men and morning salivary cortisol in women and men were not associated with the risk of T2DM development (22). Thus, both studies showed that overall morning or evening salivary cortisol do not predict new onset of T2DM $(22,23)$, which is in line with our results. Therefore, although salivary cortisol has been shown to be a marker of the HPA axis (14), it does not predict incident IR or T2DM, suggesting that stress of the HPA axis might not be the cause, but could be secondary to T2DM, as proposed by several studies $(15,18)$.

\section{Strengths and limitations}

Strengths in the present study include a relatively large sample size of healthy adults and the homogeneity of the

Table 5 Distribution between quartiles of evening salivary cortisol ( $\mathrm{nmol} / \mathrm{L})$ of healthy participants who developed $(n=359)$ and who remained free $(n=1115)$ from insulin resistance ${ }^{a}$ and remained free $(n=1444)$ and developed diabetes mellitus $(n=30)$ at 5.3 years average follow-up ${ }^{\mathrm{b}}$.

\begin{tabular}{|c|c|c|c|c|c|}
\hline & First & Second & Third & Fourth & $P$ value \\
\hline Salivary cortisol, median (range) & $1.6(0.3-2.0)$ & $2.4(2.0-3.0)$ & $3.5(3.0-4.3)$ & $5.9(4.3-41.8)$ & 0.082 \\
\hline No insulin resistance, $n(\%)$ & $282(76.4)$ & $292(79.4)$ & $263(71.3)$ & $278(75.5)$ & \\
\hline Incident insulin resistance, $n(\%)$ & $87(23.6)$ & $76(20.7)$ & $106(28.7)$ & $90(24.5)$ & \\
\hline Salivary cortisol, median (range) & $1.6(0.3-2.0)$ & $2.4(2.0-3.0)$ & $3.5(3.0-4.3)$ & $5.9(4.3-41.8)$ & 0.510 \\
\hline No diabetes mellitus, $n(\%)$ & $364(98.6)$ & $359(97.6)$ & $359(97.3)$ & $362(98.4)$ & \\
\hline Diabetes mellitus, $n(\%)$ & $5(1.4)$ & $9(2.5)$ & $10(2.7)$ & $6(1.6)$ & \\
\hline
\end{tabular}

Statistical analysis by chi-square; the $P$ value corresponds to the chi-square comparing the distribution of participants with and without incident insulin resistance between quartiles of the different variables. $n=1444$ no diabetes mellitus and $n=30$ diabetes mellitus.

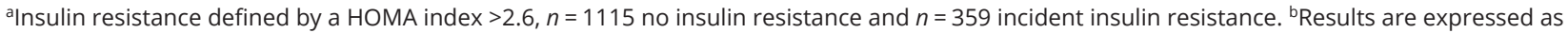
number of participants (percentage).

https://ec.bioscientifica.com https://doi.org/10.1530/EC-19-0251 (c) 2019 The authors Published by Bioscientifica Ltd

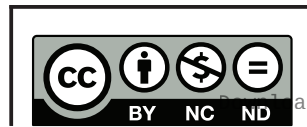

This work is licensed under a Creative Commons Attribution-NonCommercial-NoDerivatives 4.0 Internationab bicense.ifica.com at 04/26/2023 02:12:10AM 
Table 6 Multivariable analysis of the association between quartiles of evening salivary cortisol and incident insulin resistance and diabetes mellitus at 5.3 years average follow-up.

Incident insulin resistance
Cases (\%)
Multivariable model 1
Multivariable model 2
Diabetes mellitus
Cases (\%)
Multivariable model 1
Multivariable model 2

\begin{tabular}{c}
\hline First \\
\hline 87 (23.6) \\
1 (ref) \\
1 (ref) \\
$5(1.4)$ \\
1 (ref) \\
1 (ref) \\
\hline
\end{tabular}

\begin{tabular}{c}
\hline Second \\
\hline $76(20.7)$ \\
$0.83(0.58-1.19)$ \\
$0.83(0.57-1.23)$ \\
$9(2.5)$ \\
$1.75(0.58-5.31)$ \\
$1.74(0.57-5.31)$ \\
\hline
\end{tabular}

\begin{tabular}{c} 
Third \\
\hline $106(28.7)$ \\
$1.27(0.91-1.78)$ \\
$1.27(0.87-1.84)$ \\
$10(2.7)$ \\
$1.89(0.63-5.62)$ \\
$1.78(0.59-5.37)$ \\
\hline
\end{tabular}

\begin{tabular}{c}
\hline Fourth \\
\hline $90(24.5)$ \\
$0.95(0.68-1.34)$ \\
$0.95(0.65-1.40)$ \\
$6(1.6)$ \\
$1.05(0.31-3.50)$ \\
$1.00(0.29-3.37)$ \\
\hline
\end{tabular}

\begin{tabular}{l} 
P value \\
\hline \\
\\
0.610 \\
0.655 \\
\\
\\
\\
0.911 \\
0.995 \\
\hline
\end{tabular}

Insulin resistance was defined by a HOMA index $>2.6 . n=1444$ no diabetes mellitus and $n=30$ diabetes mellitus. Statistical analysis was conducted by logistic regression and results are expressed as odds ratio ( $95 \%$ confidence interval). Multivariable model 1: adjusting on age and gender. Multivariable model 2: adjusting on age, gender, BMI, physical activity and smoking status.

$n=1115$ no insulin resistance and $n=359$ incident insulin resistance.

population. We used incident IR using a validated cut-off for the HOMA index (28) not used in the previous study assessing salivary cortisol and risk of new T2DM (23). Nevertheless, our study has some limitations. First, there is a substantial amount of individuals that were not analyzed (69.9\%), mainly because of the absence of salivary cortisol measurements. Still, inverse probability weighting taking into account the likelihood of being included led to similar findings. The relatively short follow-up (5.3 y) reduced the amount of new-onset IR or T2DM, whose incidence was low, and thus reduced statistical power. Moreover, due to the nature of our epidemiological study, diagnosis of T2DM was based only on fasting plasma glucose and not on an oral glucose tolerance test or HbA1c, which may lead to an underestimation of incident IR or T2DM cases. Several factors may impact cortisol measurement, such as oral, injected or topical glucocorticoids. Nevertheless, our analysis shows that there was no significant difference between treated and untreated subjects by corticoids regarding incident IR or T2DM (data not shown). Also, estrogen replacement or contraceptive pills are known to affect total cortisol levels. However, these drugs do not affect salivary cortisol, which measures free cortisol. Exact time of morning and evening salivary cortisol collection was not reported in our study and thus time between awaking and cortisol measurement may vary between participants. Also, due to the nature of the study, we could not measure free urinary cortisol, ACTH or cortisolemia, which might be better hormonal markers than salivary cortisol. Finally, this study included only Caucasian participants, generalization of the results should therefore be cautious.

In conclusion, our study suggests that basal and evening salivary cortisol measurements, in a cohort of healthy adults of a wide age range, are not associated with incident IR or T2DM, even after adjustment for the main risk factors pertaining to IR and T2DM.
Supplementary data

This is linked to the online version of the paper at https://doi.org/10.1530/ EC-19-0251.

\section{Declaration of interest}

The authors declare that there is no conflict of interest that could be perceived as prejudicing the impartiality of the research reported.

\section{Funding}

The CoLaus/PsyCoLaus study was and is supported by research grants from GlaxoSmithKline, the Faculty of Biology and Medicine of Lausanne, and the Swiss National Science Foundation (grants 33CSCO-122661, 33CS30-139468 and 33CS30-148401).

\section{Author contribution statement}

K G analyzed data and wrote part of the manuscript; P M V analyzed data and wrote part of the manuscript; G W performed the experiments, reviewed/edited manuscript and contributed to discussion; P V performed the experiments, reviewed/edited manuscript and contributed to discussion; F R J analyzed data and wrote part of the manuscript. P M V had full access to the data and is the guarantor of the study.

\section{References}

1 Clayton RN, Raskauskiene D, Reulen RC \& Jones PW. Mortality and morbidity in Cushing's disease over 50 years in Stoke-on-Trent, UK: audit and meta-analysis of literature. Journal of Clinical Endocrinology and Metabolism 201196 632-642. (https://doi.org/10.1210/jc.2010-1942)

2 Bjorntorp P, Holm G \& Rosmond R. Hypothalamic arousal, insulin resistance and type 2 diabetes mellitus. Diabetic Medicine 199916 373-383. (https://doi.org/10.1046/j.1464-5491.1999.00067.x)

3 Andrews RC, Herlihy O, Livingstone DEW, Andrew R \& Walker BR. Abnormal cortisol metabolism and tissue sensitivity to cortisol in patients with glucose intolerance. Journal of Clinical Endocrinology and Metabolism 200287 5587-5593. (https://doi.org/10.1210/jc.2002020048)

4 Gozansky WS, Lynn JS, Laudenslager ML \& Kohrt WM. Salivary cortisol determined by enzyme immunoassay is preferable to serum total cortisol for assessment of dynamic hypothalamic-pituitaryadrenal axis activity. Clinical Endocrinology 200563 336-341. (https:// doi.org/10.1111/j.1365-2265.2005.02349.x)

5 Anagnostis P, Athyros VG, Tziomalos K, Karagiannis A \& Mikhailidis DP. Clinical review: The pathogenetic role of cortisol 
in the metabolic syndrome: a hypothesis. Journal of Clinical Endocrinology and Metabolism 200994 2692-2701. (https://doi. org/10.1210/jc.2009-0370)

6 Bruehl H, Rueger M, Dziobek I, Sweat V, Tirsi A, Javier E, Arentoft A, Wolf OT \& Convit A. Hypothalamic-pituitary-adrenal axis dysregulation and memory impairments in type 2 diabetes. Journal of Clinical Endocrinology and Metabolism 200792 2439-2445. (https:// doi.org/10.1210/jc.2006-2540)

7 Chiodini I, Torlontano M, Scillitani A, Arosio M, Bacci S, Di Lembo S, Epaminonda P, Augello G, Enrini R, Ambrosi B, et al. Association of subclinical hypercortisolism with type 2 diabetes mellitus: a casecontrol study in hospitalized patients. European Journal of Endocrinology 2005153 837-844. (https://doi.org/10.1530/eje.1.02045)

8 Godoy-Matos AF, Vieira AR, Moreira RO, Coutinho WF, Carraro LM, Moreira DM, Pasquali R \& Meirelles RM. The potential role of increased adrenal volume in the pathophysiology of obesity-related type 2 diabetes. Journal of Endocrinological Investigation 200629 159-163. (https://doi.org/10.1007/BF03344090)

9 Champaneri S, Xu X, Carnethon MR, Bertoni AG, Seeman T, DeSantis AS, Diez Roux AD, Shrager S \& Golden SH. Diurnal salivary cortisol is associated with body mass index and waist circumference: the multiethnic study of atherosclerosis. Obesity 201321 E56-E63. (https://doi.org/10.1002/oby.20047)

10 Gagliardi L, Chapman IM, O’Loughlin P \& Torpy DJ. Screening for subclinical Cushing's syndrome in type 2 diabetes mellitus: low falsepositive rates with nocturnal salivary cortisol. Hormone and Metabolic Research 201042 280-284. (https://doi.org/10.1055/s-0029-1246191)

11 Muscogiuri G, Sorice GP, Prioletta A, Mezza T, Cipolla C, Salomone E, Giaccari A, Pontecorvi A \& Della Casa S. The size of adrenal incidentalomas correlates with insulin resistance. Is there a causeeffect relationship? Clinical Endocrinology 201174 300-305. (https:// doi.org/10.1111/j.1365-2265.2010.03928.x)

12 Tsuiki M, Tanabe A, Takagi S, Naruse M \& Takano K. Cardiovascular risks and their long-term clinical outcome in patients with subclinical Cushing's syndrome. Endocrine Journal 200855 737-745. (https://doi.org/10.1507/endocrj.K07E-177)

13 Pivonello R, Faggiano A, Lombardi G \& Colao A. The metabolic syndrome and cardiovascular risk in Cushing's syndrome. Endocrinology and Metabolism Clinics of North America 200534 327-339. (https://doi.org/10.1016/j.ecl.2005.01.010)

14 Siddiqui A, Madhu SV, Sharma SB \& Desai NG. Endocrine stress responses and risk of type 2 diabetes mellitus. Stress $2015 \mathbf{1 8}$ 498-506. (https://doi.org/10.3109/10253890.2015.1067677)

15 Virtanen M, Ferrie JE, Tabak AG, Akbaraly TN, Vahtera J, SinghManoux A \& Kivimäki M. Psychological distress and incidence of type 2 diabetes in high-risk and low-risk populations: the Whitehall II Cohort Study. Diabetes Care 201437 2091-2097. (https://doi. org/10.2337/dc13-2725)

16 Hellhammer DH, Wust S \& Kudielka BM. Salivary cortisol as a biomarker in stress research. Psychoneuroendocrinology 200934 163-171. (https://doi.org/10.1016/j.psyneuen.2008.10.026)

17 Rosmond R. Stress induced disturbances of the HPA axis: a pathway to type 2 diabetes? Medical Science Monitor 20039 RA35-RA39. (https://doi.org/10.2337/dc19-S002)

18 Hackett RA, Steptoe A \& Kumari M. Association of diurnal patterns in salivary cortisol with type 2 diabetes in the Whitehall II study. Journal of Clinical Endocrinology and Metabolism 201499 4625-4631. (https://doi.org/10.1210/jc.2014-2459)

19 Chiodini I, Adda G, Scillitani A, Coletti F, Morelli V, Di Lembo S, Epaminoda P, Masserini B, Beck-Peccoz P, Orsi E, et al. Cortisol secretion in patients with type 2 diabetes: relationship with

chronic complications. Diabetes Care 200730 83-88. (https://doi. org/10.2337/dc06-1267)

20 Debono M, Ghobadi C, Rostami-Hodjegan A, Huatan H, Campbell MJ, Newell-Price J, Darzy K, Merke DP, Arlt W \& Ross RJ. Modified-release hydrocortisone to provide circadian cortisol profiles. Journal of Clinical Endocrinology and Metabolism 200994 1548-1554. (https://doi.org/10.1210/jc.2008-2380)

21 Viardot A, Huber P, Puder JJ, Zulewski H, Keller U \& Muller B. Reproducibility of nighttime salivary cortisol and its use in the diagnosis of hypercortisolism compared with urinary free cortisol and overnight dexamethasone suppression test. Journal of Clinical Endocrinology and Metabolism 200590 5730-5736. (https://doi.org/10.1210/jc.2004-2264)

22 Schoorlemmer RMM, Peeters GMEE, van Schoor NM \& Lips P. Relationships between cortisol level, mortality and chronic diseases in older persons. Clinical Endocrinology 200971 779-786. (https:// doi.org/10.1111/j.1365-2265.2009.03552.x)

23 Hackett RA, Kivimaki M, Kumari M \& Steptoe A. Diurnal cortisol patterns, future diabetes, and impaired glucose metabolism in the Whitehall II cohort study. Journal of Clinical Endocrinology and Metabolism 2016101 619-625. (https://doi.org/10.1210/jc.2015-2853)

24 Firmann M, Mayor V, Vidal PM, Bochud M, Pecoud A, Hayoz D, Paccaud F, Preisig M, Song KS, Yuan X, et al. The CoLaus study: a population-based study to investigate the epidemiology and genetic determinants of cardiovascular risk factors and metabolic syndrome. BMC Cardiovascular Disorders $2008 \mathbf{8}$ 6. (https://doi. org/10.1186/1471-2261-8-6)

25 Jornayvaz FR, Vollenweider P, Bochud M, Mooser V, Waeber G \& Marques-Vidal P. Low birth weight leads to obesity, diabetes and increased leptin levels in adults: the CoLaus study. Cardiovascular Diabetology 201615 73. (https://doi.org/10.1186/s12933-016-0389-2)

26 Kuehner C, Holzhauer S \& Huffziger S. Decreased cortisol response to awakening is associated with cognitive vulnerability to depression in a nonclinical sample of young adults. Psychoneuroendocrinology 2007 32 199-209. (https://doi.org/10.1016/j.psyneuen.2006.12.007)

27 American Diabetes Association. 2. Classification and diagnosis of diabetes: standards of medical care in diabetes-2019. Diabetes Care 201942 (Supplement 1) S13-S28

28 Matthews DR, Hosker JP, Rudenski AS, Naylor BA, Treacher DF \& Turner RC. Homeostasis model assessment: insulin resistance and beta-cell function from fasting plasma glucose and insulin concentrations in man. Diabetologia 198528 412-419. (https://doi. org/10.1007/BF00280883)

29 Marques-Vidal P, Vollenweider P, Guessous I, Henry H, Boulat O, Waeber G \& Jornayvaz FR. Serum vitamin D concentrations are not associated with insulin resistance in Swiss adults. Journal of Nutrition 2015145 2117-2122. (https://doi.org/10.3945/jn.115.211763)

30 Bernstein M, Sloutskis D, Kumanyika S, Sparti A, Schutz Y \& Morabia A. Data-based approach for developing a physical activity frequency questionnaire. American Journal of Epidemiology 1998147 147-154. (https://doi.org/10.1093/oxfordjournals.aje.a009427)

31 Narduzzi S, Golini MN, Porta D, Stafoggia M \& Forastiere F. Inverse probability weighting (IPW) for evaluating and 'correcting' selection bias. Epidemiologia e Prevenzione 201438 335-341.

32 Clow A, Hucklebridge F \& Thorn L. The cortisol awakening response in context. International Review of Neurobiology 201093 153-175. (https://doi.org/10.1016/S0074-7742(10)93007-9)

33 Hellhammer J, Fries E, Schweisthal OW, Schlotz W, Stone AA \& Hagemann D. Several daily measurements are necessary to reliably assess the cortisol rise after awakening: state- and trait components. Psychoneuroendocrinology 200732 80-86. (https://doi.org/10.1016/j. psyneuen.2006.10.005)

Received in final form 24 May 2019

Accepted 6 June 2019

Accepted Preprint published online 6 June 2019

https://ec.bioscientifica.com https://doi.org/10.1530/EC-19-0251 (c) 2019 The authors Published by Bioscientifica Ltd

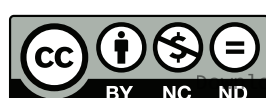

This work is licensed under a Creative Commons Attribution-NonCommercial-NoDerivatives 4.0 Internationab bicense.ifica com at 04/26/2023 02:12:10AM 